\title{
EP332: A Novel Clinical Phenotype of Acquired Partial Lipodystrophy Associated with Intensive Childhood Cytostatic Treatment
}

\section{Introduction}

- Lipodystrophy is a rare clinical syndrome characterized by subcutaneous fat loss, metabolic syndrome and fat maldistribution

- Common causes are HIV therapy, specific genetic mutations and autoimmune disease

- Partial lipodystrophy of the limbs with severe insulin resistance has been reported ${ }^{2}$

\section{Methods}

- Detailed description of two cases with this specific phenotype

- Both patients were referred for treatment refractory type 2 diabetes

\section{Patient 1: Case history}

- 43 year old Caucasian female - Treated with intensive polychemotherapy for leukemic lymphosarcoma at age 6 through 13

Presented with treatment resistant diabetes, hypertension and dyslipidemia Complaints: central fat deposition, high glucose levels

Current therapy: atorvastatine, tolbutamide, lantus in increasing dose Physical: BP 170/100 mmHg, bmi 23 $\mathrm{kg} / \mathrm{m}^{2}$; Notable excess fat deposition at face, trunk, upper arms. Lipoatrophy of hips and distal extremities

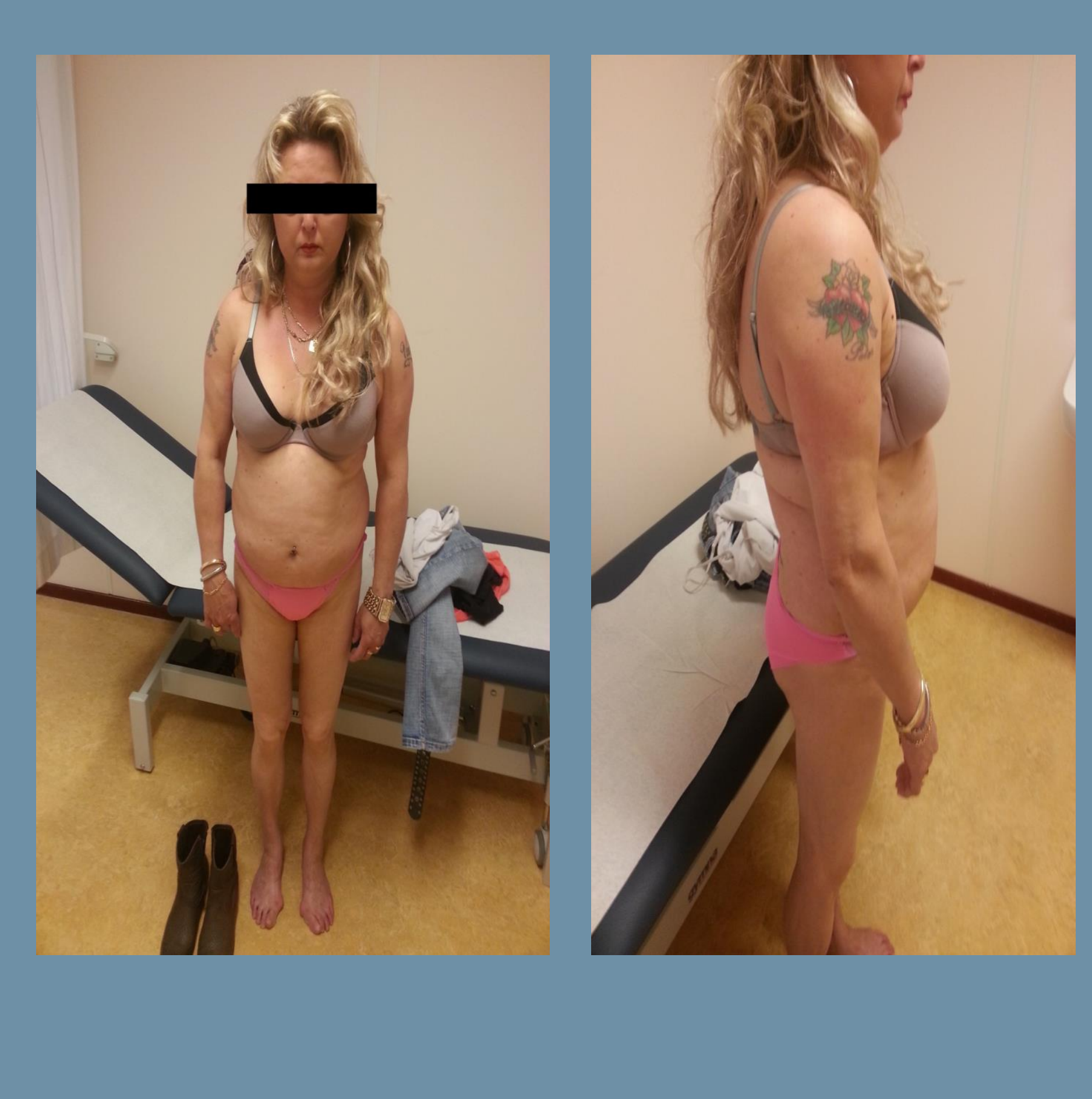

Patient 1: Relevant laboratory results

\begin{tabular}{|l|l|l|l|}
\hline & Before pioglitazone & After pioglitazone & Reference values \\
\hline ALAT & $\mathbf{4 2}$ & 26 & $<34 \mathrm{U} / \mathrm{L}$ \\
\hline Gamma GT & $\mathbf{1 1 8}$ & $\mathbf{5 1}$ & $<38 \mathrm{U} / \mathrm{L}$ \\
\hline Triglycerides & $1.8^{*}$ & $=$ & $<2.30 \mathrm{mmol} / \mathrm{L}$ \\
\hline HbA1c & $\mathbf{6 6}$ & 36 & $<42 \mathrm{mmol} / \mathrm{mol} \mathrm{Hb}$ \\
\hline Fasting glucose & $\mathbf{9 . 3}$ & 5.1 & $3.1-6.0 \mathrm{mmol} / \mathrm{L}$ \\
\hline C-peptide & $\mathbf{4 . 1}$ & $\mathbf{2 . 1}$ & $0.3-1.3 \mathrm{nmol} / \mathrm{L}$ \\
\hline Leptin & $\mathbf{3 5 . 1}$ & $\mathbf{5 5 . 3}$ & $3.7-11.1 \mathrm{mg} / \mathrm{L}$ \\
\hline
\end{tabular}

\section{* While taking high dose statin therapy}

Patient 1: Clinical course

After initiation of pioglitazone

$30 \mathrm{mg}$ once daily, blood pressure

glucose metabolism and liver

enzymes normalized.

No change in fat maldistribution

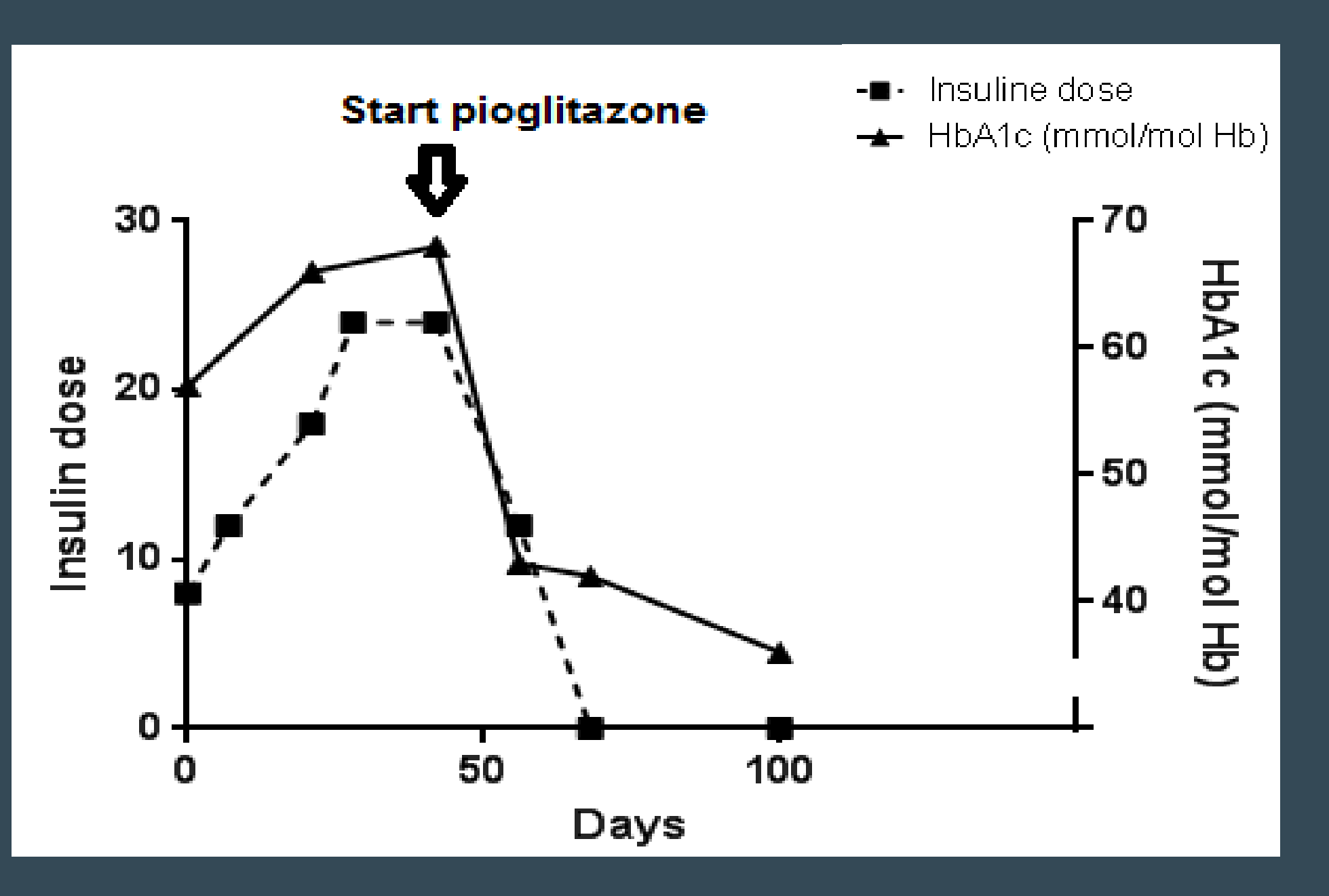

\section{AIM: To report on a novel phenotype of partial acquired lipodystrophy with severe insulin resistance and elevated leptin levels, associated with intensive cytotoxic treatment in childhood \\ - Both had received intensive cytotoxic treatment in childhood \\ - Genetic and auto-immune testing was negative, and no underlying endocrine disorder was identified (i.e. Cushing's syndrome, lipid metabolism disorders) \\ - Treatment with pioglitazone was initiated}

\section{Patient 2: Case history}

- 22 year old Caucasian female

Treated with high dose cyclophosphamide and total body irradiation for aplastic anemia at age 12

Presented with treatment resistant diabetes, hypertriglyceridemia

Complaints: recurrent graft-versus-host of the skin. High glucose levels despite metformin. Central fat deposition

Current therapy: metformin, s.c. insulin in - increasing dose

Physical: BP 160/90 mmHg, bmi $22 \mathrm{~kg} / \mathrm{m}^{2}$ Severe fibrous skin scarring due to GvHD, notable lipoatrophy of extremities and hips, excess fat deposition at the trunk

\section{Patient 2: Relevant laboratory results}

\begin{tabular}{|l|l|l|l|}
\hline & Before pioglitazone & After pioglitazone & Reference values \\
\hline ALAT & $\mathbf{7 4}$ & $\mathbf{4 7}$ & $<34 \mathrm{U} / \mathrm{L}$ \\
\hline Gamma GT & $\mathbf{2 2 2}$ & $\mathbf{7 0}$ & $<38 \mathrm{U} / \mathrm{L}$ \\
\hline Triglycerides & $\mathbf{2 9 . 9}$ & $\mathbf{6 , 8}$ & $<2.30 \mathrm{mmol} / \mathrm{L}$ \\
\hline HbA1c & $\mathbf{5 2 ^ { * }}$ & $34^{*}$ & $<42 \mathrm{mmol} / \mathrm{mol} \mathrm{Hb}$ \\
\hline Fasting glucose & $\mathbf{1 0 , 9}$ & 4.6 & $3.1-6.0 \mathrm{mmol} / \mathrm{L}$ \\
\hline C-peptide & $\mathbf{2 . 0}$ & $\mathrm{ND}$ & $0.3-1.3 \mathrm{nmol} / \mathrm{L}$ \\
\hline Leptin & $\mathbf{1 5 . 5}$ & $\mathrm{ND}$ & $3.7-11.1 \mathrm{\mu g} / \mathrm{L}$
\end{tabular}

\section{Patient 2: Clinical course}

After careful initation of pioglitazone $30 \mathrm{mg}$ once daily, insulin requirement decreased dramatically. Also, blood pressure, liver enzymes and triglycerides improved. No change in fat maldistribution
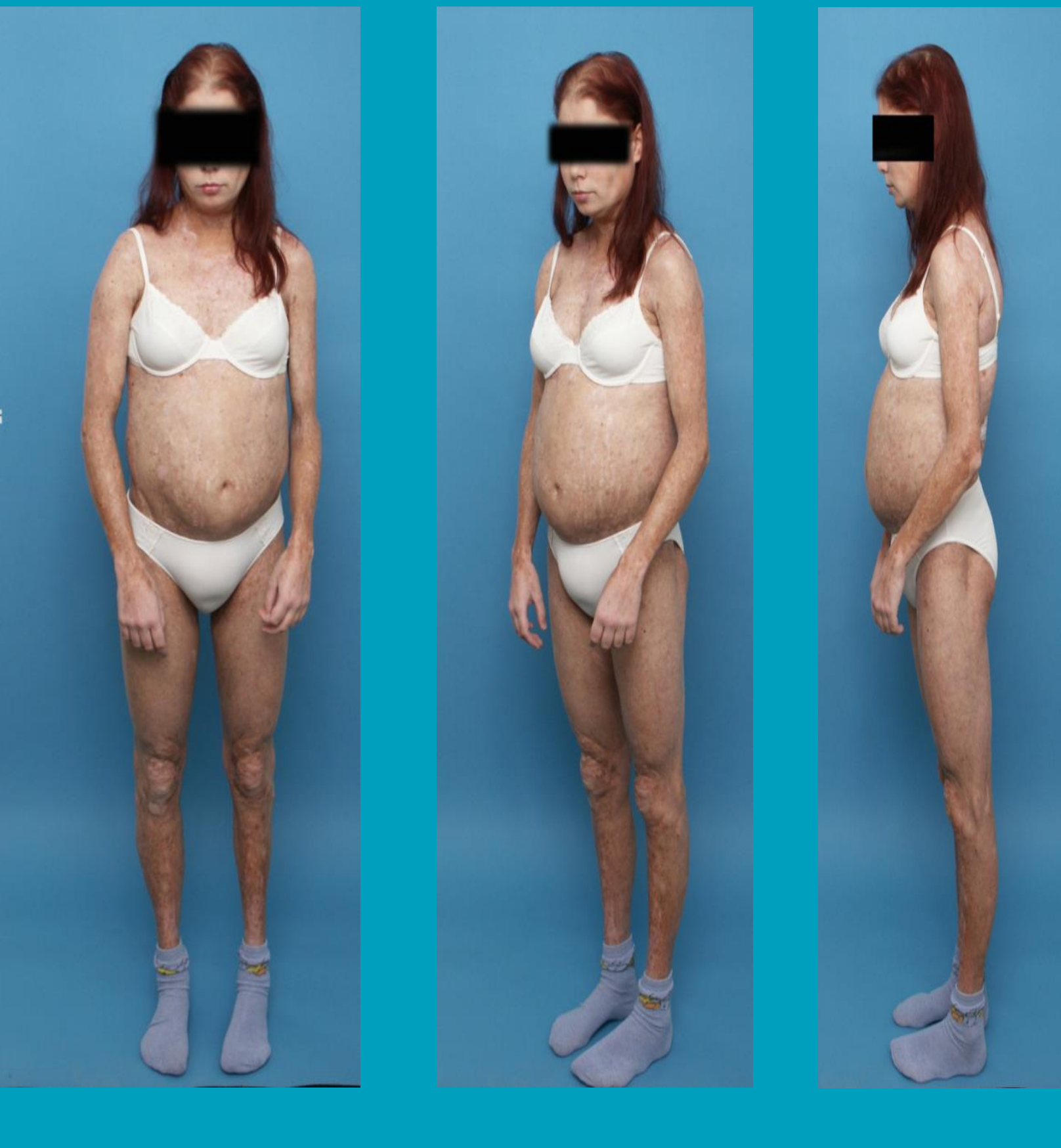

\section{Conclusions}

- Acquired partial lipodystrophy can be associated with intensive cytostatic treatment in childhood.

- This phenotype, characterized by loss of subcutanous fat at the extremities and buttocks in the presence of elevated leptin levels, did not match previously reported types of lipodystrophy.

- Pioglitazone treatment appears to be particularly effective at treating the specific associated metabolic disorders.

\section{References}

1) Garg A.. J Clin Endocrinol Metab 2011

2) Strickland LR et al. Diabetes Care 2013

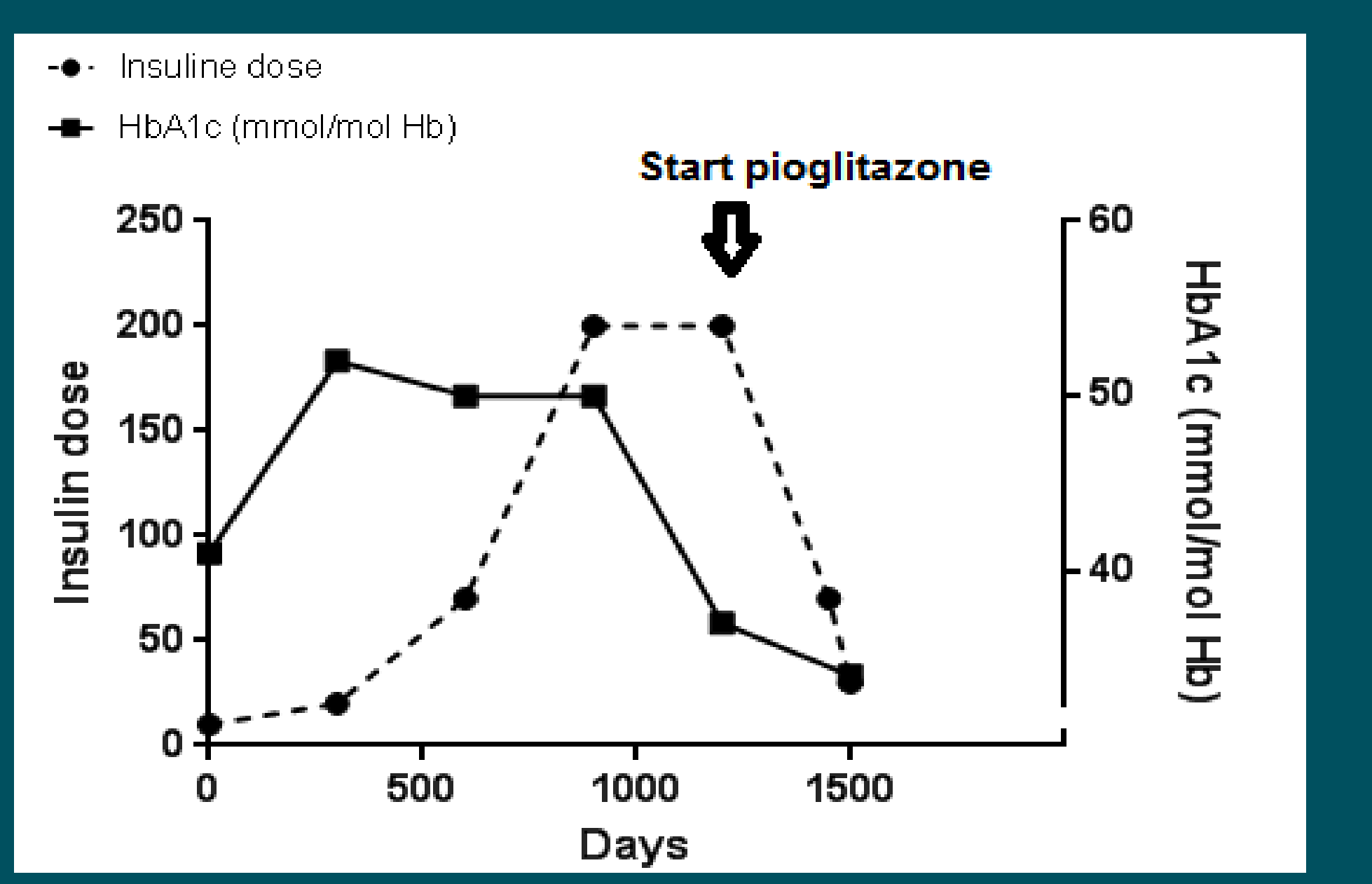

